\title{
Tibetan wild asses (Equus Kiang) were superior to domestic donkeys (Equus Asinus) in terms of the composition and function of gut microbial community
}

Hongjin Liu

Northwest Institute of Plateau Biology Chinese Academy of Sciences

Xinquan Zhao

Northwest Institute of Plateau Biology Chinese Academy of Sciences

Shixiao Xu ( $\nabla$ sxxu@nwipb.cas.cn)

Northwest Institute of Plateau Biology Chinese Academy of Sciences

Liang Zhao

Northwest Institute of Plateau Biology Chinese Academy of Sciences

\section{Linyong $\mathrm{Hu}$}

Northwest Institute of Plateau Biology Chinese Academy of Sciences

Tianwei Xu

Northwest Institute of Plateau Biology Chinese Academy of Sciences

Na Zhao

Northwest Institute of Plateau Biology Chinese Academy of Sciences

Xiaoling Zhang

Northwest Institute of Plateau Biology Chinese Academy of Sciences

Qi Li

Northwest Institute of Plateau Biology Chinese Academy of Sciences

\section{Dongdong Chen}

Institute of Tibetan Plateau Research Chinese Academy of Sciences

\section{Fuquan $\mathrm{He}$}

Northwest Institute of Plateau Biology Chinese Academy of Sciences

Xin Chen

Northwest Institute of Plateau Biology Chinese Academy of Sciences

\section{Research article}

Keywords: Tibetan wild asses, domestic donkeys, 16 S ribosomal RNA gene, gut microbiota

Posted Date: September 10th, 2019

DOI: https://doi.org/10.21203/rs.2.14192/v1

License: (c) (i) This work is licensed under a Creative Commons Attribution 4.0 International License. Read Full License 


\section{Abstract}

Background: Tibetan wild asses are the only wild species of perissodactyls on the Qinghai-Tibetan Plateau, and appears on the International Union for Conversation of Nature (IUCN) 2012 Red List of threatened species. The gut microbiota has a great effect on the health and nutrition of the host, however, scant research is available on the characteristics of their intestinal microbiota. Therefor, understanding the gut microbita composition and function of TWAs can provide a theoretical for the situ conservation of wild animals in the future.

Results: To characterize its composition and function, we analyzed the intestinal microbiota of wild asses and domestic donkeys by high-throughput sequencing of the $16 \mathrm{~s}$ rDNA regions. No significant difference in alpha diversity was detected between these two groups. Beta diversity showed that the bacterial community structure of wild asses was acutely different from domestic donkeys. At the phylum level, the two dominant phyla of Bacteroidetes and Firmcutes in wild asses were significantly higher than that in domestic donkeys. At the genus level, Ruminococcaceae_NK4A214 , Phascolarctobacterium , Coprostanoligenes_group , Lachnospiraceae_XPB1014_group and Akkermansia in wild asses were significantly higher than domestic donkeys. Moreover, statistical comparisons showed that 40 different metabolic pathways exhibited significant differences . Among them, 29 pathways had richer concentrations in wild asses than domestic donkeys, mainly amino acid metabolism, carbohydrate metabolism, and energy metabolism. Of note, network analysis showed that wild asses harbored a relatively more complex bacterial network than domestic donkeys, possibly reflecting the specific niche adaption of gut bacterial communities through species interactions.

Conclusions: Wild asses were superior to that of domestic in gut microbial community composition and function. For wild animal conservation, wild asses are more suitable to survive in wild than to be domesticated or captive.

Key words: Tibetan wild asses, domestic donkeys, 16S ribosomal RNA gene, gut microbiota

\section{Background}

Low temperatures and hypoxia render the Qinghai-Tibetan Plateau (QTP) an extremely harsh environment for the survival of mammalian species. The Tibetan wild asses (Equus kiang)_order Perissodactyla, family Equidae, genus Equus-is considered a unique species and the only wild species of perissodactyls to inhabit the QTP [1], and appears on the International Union for Conversation of Nature (IUCN) 2012 Red List of threatened species. Extensive research has been conducted to promote the conservation of this species [2, 3]. Domestic donkeys (Equus asinus) —also of the order Perissodactyla, family Equidae, genus Equus-are the subspecies of wild ass [4] and were introduced from regions of China at lower elevations, adapting to life in this harsh environment and becoming the livestock of the nomadic Tibetan people to their substantial economic benefit (meat and skin). The intestines of Tibetan wild asses (TWAs) and domestic donkeys contain complex microorganisms that play an important role in converting indigestible plants into the energy needed for host growth, serving a bridge between forage digestion and host energy absorption. Therefore, the study of intestinal microorganisms is of great significance for revealing host immunity, nutrient metabolism, energy absorption and wild animal conservation.

Numerous scientific reports suggest that gut microbiota constitute a complex ecosystem involving a symbiotic relationship with the host that plays an important role in the host's performance, health and disease, nutrient absorption, and metabolic status [5-8]. To date, this research has focused mainly on humans, ruminants, marine organisms, and some monogastric animals to investigate the factors that affect intestinal microbiota, gut microbes and health, and gut microbes and nutritional metabolism $[5,9,10]$. As herbivores, donkeys utilize forage nutrition by 
microorganism fermentation in the gastrointestinal tract. Zhao et al. (2016) studied bacterial diversity in the ceca of Xinjiang donkeys and found that Ruminococcaceae and Lachnospiraceae played a key role in digesting roughage feed [11]. Due to the difficulty of obtaining samples from wild protected animals, research on gut microbes in TWAs is limited. A recent study compared the gut microbiota composition of wild and captive TWAs, revealing that captivity reduced intestinal microbial diversity and increased the risk of epidemics, thereby negatively impacting the health of wild life [12]. However, few studies have compared the intestinal microbial composition and function of TWAs and natural pasture domestic donkeys (NPDDs) on the QTP.

As members of the same genus-Equus-",TWAs and NPDDs provide ideal candidates for the study of gut microbiota. Therefore, we used high-throughput sequencing of the V3-V4 regions of the 16s rRNA gene to investigate the composition and predict the metabolic pathways and functions of the gut microbes in TWAs and NPDDs. We hypothesized that TWAs were superior to NPDDs in terms of the composition and function of their intestinal flora. We think our research is of great significance to wildlife conservation.

\section{Results}

\section{Analysis of food nutrition composition between TWAs and NPDDs}

As shown in Table 1, themainlyplant-based diet for TWAs were Kobresia tibetica and Kobresia pygmaea, while for NPDDs were Kobresia humilis and Kobresia capillifolia. There was no significant difference in nutrient composition except ash content $(P<0.05)$.

\section{Sequencing and classification}

Illumina sequencing yielded 2540672 raw reads of 16 S gene sequences. After quality filtering, a total of 2395867 effective tags were obtained (Table S1). As shown in Fig. S1, 12923 operational taxonomic units (OTUs) at a cutoff of $97 \%$ similarity were generated. Among these OTUs, the number of unique OTUs in TWAs and NPDDs was 2948 and 2816 , respectively, and 7159 OTUs were shared by all samples, accounting for $55.40 \%$ of the total OTUs.

\section{Diversity analysis of gut microbiota in TWAs and NPDDs}

To determine alpha diversity, we calculated the Chao1, ACE, observed species estimates and the Shannon diversity index. As shown in Fig. 1,no significant differences were found in these two groups for the Shannon index, Chao1, ACE estimates and the number of observed species. The OTU level rarefaction curves of diversity estimators reached a plateau at the minimum sequence number of 50,000 tags (Fig. S2).. With regard to beta diversity, PCoA analysis showed that the two groups of bacterial communities differed significantly (Fig. S3A) $(P<0.05)$. To assess the overall similarity, UPGAMA with classification tree analysis was conducted as shown in Fig. S3B. It is obvious that the bacterial community of TWAs was completely different from that of NPDDs.

\section{Significance analysis of gut bacterial community abundance between TWAs and NPDDs}

At the phylum level illustrated in Fig. 2A, Bacteroidetes, Firmicutes, Verrucomicrobia, and Fibrobacteres constituted the four dominant phyla in all samples. The average ratio of Firmicutes/Bacteroidetes was 0.80 and 0.74 , 
respectively. The significance analysis of the top 10 phyla of microbial communities between TWAs and NPDDs is shown in Fig. 2B. The relative abundance of Bacteroidetes, Firmicutes, Cyanobacteria, and Synergistetes in TWDs was significantly higher than in NPDDs, whereas Lentisphaerae in TWAs was significantly lower $(P<0.05)$. No significant differences were detected for the relative abundances of Verrucomicrobia, Fibrobacteres, Spirochaetae, Proteobacteria, and Planctomycetes between the two groups $P>0.05$ ).

At the genus level, a total of 15 genera with proportions above $1 \%$ were detected, as shown in Fig. $3 A$. The Rickenllaceae_RC9_gut group, Fibrobacter, and Treponema_2 accounted for the largest proportions in all samples. Differentiation analysis of the 15 genera are presented in Fig. 3B. The proportion of Ruminococcaceae_NK4A214, Phascolarctobacterium, Coprostanoligenes_group, Lachnospiraceae_XPB1014_group, and Akkermansia in TWAs was significantly higher than in NPDDs, whereas the proportion of the Lachnospiraceae_AC2044_group in NPDDs was significantly higher than in TWAs $(P<0.05)$. No remarkable difference was detected in the relative abundance of the Rickenellaceae_RC9_gut_group, Fibrobacter, Treponema, Ruminococcaceae_UCG-010,Christensenellaceae_R7_group, Prevotellaceae_UCG-001, Anawrovorax, Prevotellaceae_UCG-003, and Prevotellaceae_UCG-004 between the two groups $(P>0.05)$.

To study the main differences in gut microbiota between groups TWA and NPDD, linear discriminant analysis (LDA) combined effect size measurements (LEfSe) were used to identify the discriminative features of bacteria in the TWAs and NPDDs (Fig. S4). The taxonomic units of Campylobacter, Campylobacteraceae, Campylobacterales, Epsilonproteobacteria, andCampylobacter_hyointestinalis_subsp_hyointestinalis, which belong to the phylum Proteobacteria, were significantly enriched and revealed different biomarkers in TWAs, whereas no specific taxonomic units was enriched in NPDD.

\section{Predicted metabolic pathways and functions of the bacterial microbiota between TWAs and NPDDs}

A total of six types of biological functional pathways in KEGG level 1 were detected (Fig. 4A).The metabolism pathways had the highest relative abundance, with more than $60 \%$ of the total reads in each group. At KEGG level 2 , a total of 37 metabolic pathways were detected in the gut samples (Table S2).. As shown in Fig. 4B, 18 gene families were remarkably different and the relative abundance of amino acid metabolism, energy metabolism, glycan biosynthesis, and the biosynthesis of other secondary metabolites in TWAs were significantly higher than in NPDDs $(P<0.05)$. As for membrane transport and cell motility, NPDDs had higher expression abundances than TWAs $(P<$ 0.05). At KEGG level 3, the principal component analysis and partial least squares discriminant analysis revealed clear differences between the two groups (Fig. 5). As shown in Table S3, 40 metabolite pathways were significantly different (VIP $>1$ and $P<0.05$ ), of which ten metabolites pathways belonging to amino acid metabolism were enriched in TWAs $(P<0.05)$. A total of ten metabolic pathways belonged to carbohydrate metabolism of which seven metaboliteswere enriched in TWAs, whereas the remaining three metabolites (starch and sucrose metabolism, amino sugar and nucleotide sugar metabolism, and lipopolysaccharide biosynthesis) were enriched in NPDDs $(P<$ 0.05). In addition, five metabolic pathways belonged to metabolism of cofactors and vitamins, of which two metabolites were higher in TWAs. For the remaining 15 metabolic pathways, 11 had a higher relative abundance in TWAs. 


\section{Network analysis of bacterial communities and putative keystone taxa}

As shown in Fig. 6, when using the same threshold (0.76), a total of 207 and 104 links were identified for TWAs and NPDDs. Notably, for TWAs, the percentage of positive correlations (149 out of 207) was the dominant, while negative correlations (55 out of 104) were the predominant in NPDDs. Interestingly, we found the values of total links, avgK and D were higher in TWAs than those in NPDDs, indicating a relatively simpler network in NPDDs. The lower avgCC in NPDD indicated that the bacterial network was mainly loose node groups. Moreover, TWA had a higher level of CB than NPDD, implying that TWA harbored a higher frequency of centralized.

The putative keystone genus in bacterial networks were identified based on the Pi and Zi values (Table 2). Three key module hubs and two connectors were identified in NPDD. Among these OTUs, module hubs were affiliated with Firmcutes, Cyanobacteria and unclassified bacteria, and the connectors were Anaerovibrio and unclassified bacteria. In contrast, four connector and no module hubs were identified in TWAs. Among these OTUs, two connectors were affiliated with Firmcutes and Actinobacteria and the others were the genus Oscillospira and Arthrobacter. Notably, these keystone genus in network showed relatively low abundances in gut bacterial communities.

\section{Discussion}

Although TWAs and NPDDs are herbivores species on the QTP, scant research has been conducted on their gut microbiota diversity and composition. To the best of our knowledge, this is the first study to compare the gut microbiota composition and function of TWAs and NPDDs. We found that the Shannon-Wiener index and ACE and Chao1 estimates, as well as the number of observed species between TWAs and NPDDs were not significantly different, suggesting that altitude and grassland types did not affect the alpha diversity of gut microbes. A previous study had shown that diet was one of the factors that affected gut microorganism diversity [13]. In our study, there was no significant difference in herbage nutrient composition between TWAs and NPDDs in different meadow types (swamp meadow and alpine meadow, respectively), which was consistent with the results of the alpha diversity analysis. In terms of biological classification, TWAs and NPDDs are animals of the same genus but different species; however, there was no significant difference in the alpha diversity of gut microbes, suggesting that species differences did not affect the alpha diversity of bacteria community, as was also found in a previous study on wild and domestic yak [14]. As to beta diversity, PCoA results indicated that the composition of gut microbiota was significantly different in these two groups. For a better understanding of the similarity of their gut microbiota, UPGMA methods were applied and the diagram revealed that the fecal microorganism communities in TWAs occupied their own phylogenetic tree branch which clearly distinguished from the fecal microorganisms in NPDDs. Therefore, we concluded that species, altitude, and meadow type can affect the intestinal bacterial structure; however, the determination of which of these played the leading role in shaping intestinal flora requires further studies.

To assess the composition of bacteria community, we first analyzed at the phylum level. In our study, Bacteroidetes was the most abundant phylum followed by Firmicutes in both TWAs and NPDDs, constituting more than $80 \%$ of the total bacteria content. This has been confirmed by former studies on intestinal microbial diversity in mammals [1517], as these microorganisms facilitate the digestion of cellulose and hemicellulose in the diet [18]. The relative abundance of Bacteroidetes and Firmicutes in TWAs was significantly higher than in NPDDs, indicating that the cellulose and hemicellulose decomposition capacity was stronger in TWAs than in NPDDs under similar forage nutritional composition. Moreover, the ratio of Bacteroidetes and Firmicutes affected the energy acquisition and body 
fat accumulation in humans and mice [19]. Previous studies have shown that a decrease in Bacteroidetes was strongly linked to an increase of fat in host tissue in mice and humans [19, 20]. However, scant research has focused on the relationship between gut microbiota and obesity in TWAs and NPDDs. By observing the body size of TWAs and NPDDs, we speculated that the lean body size of TWAs might be related to the high Bacteroidetes content in their intestinal microbiota. At the genus level, 15 core genera whose relative abundances were more than $1 \%$ were detected in all gut samples; these genera constituted $32.43 \%$ of the total genera reads and were mainly affiliated with the phylum Firmicutes (nine genera) and phylum Bacteroidetes (four genera). The others were affiliated with the phyla Spirochaetes (one genera) and Verrucomicrobia (one genera). These results were also reported in ruminant research on animals such as Yaks and Tibetan sheep [21, 22], indicating that although TWAs and NPDDs belonged to the genus Equus and were different from ruminants, their microorganism flora communities were actually quite similar. In addition, an average of $54.43 \%$ genera presented as unclassified, which suggested that this group of organisms may be a part of the core bacterial population, warranting further investigation in the field on Equus.

Recent studies have shown that changes in gut microbiota composition are associated with host disease [5, 23, 24]. Bacteroidetes, a basal microbe, is reported to be the most abundant phylum in healthy people [25] and captive TWAs [12]; a decrease in its relative abundance is associated with chronic diarrhea in humans [26] and could be a predictor of an animal's health. In our study, it accounted for a large proportion in TWAs (44.12\% of sequences), indicating that TWAs may have a superior capacity to stave off intestinal diseases. The phylum Spirochaetes, which contained pathogens that cause intestinal disease [27], were richer in NPDDs than in TWAs, which indicated that NPDDs would more likely suffer from intestinal diseases. At the genus level, Akkermansia accounts for the highest proportion in Verrucomicrobia. Researchers have found that Akkermansia contains a single species, namely Akkermansia muciniphila, which is an important probiotic that contributes healthy mucus-related microbiota composition $[28,29]$. In addition, Akkermansia muciniphila can be used as a probiotic to prevent obesity and type 2 diabetes $[24,30]$. In our research, TWAs had the higher distribution proportion of Akkermansia muciniphila, which may be important to the maintenance of intestinal health; however, further study it is necessary to verify this assertion.

The metabolites of gut microbiota play a pivotal role in maintaining physiologic and metabolic homeostasis in their hosts [31]. In the present study, the metagenomic function prediction showed that the two predominant intestinal microbiota in TWAs and NPDDs were related to ABC transporters and the two-component system (Table S4). Previous research $[32,33]$ has confirmed that $A B C$ transporters constitute one of the largest known protein families and are widespread in bacteria, archaea, and eukaryotes, and the two-component system is a signal transduction system that senses developmental and environmental stimuli [34]. Therefore, it is reasonable that these KOs were found to be in high abundance in the intestinal microbiota of TWAs and NPDDs. Moreover, statistical comparisons showed that 40 different metabolic pathways exhibited significant differences (Table S2). Among them, 29 pathways had richer concentrations in TWAs than NPDDs, mainly amino acid metabolism, carbohydrate metabolism, and energy metabolism. A large number of various microorganisms inhabit the intestinal tracts of monogastric animals that are symbiotic with the hosts. Gut bacteria produce a range of metabolites that provide nitrogen sources for themselves and amino acids for the hosts through the fermentation of protein and nitrogen compounds [35]. In our study, the high concentration of amino acid metabolic pathways in TWAs implied that in the context of high altitude and forage shortage, the gut microorganisms and hosts coevolved and exploited high bioavailability to efficiently utilize nitrogen compounds in forage, ultimately with microorganisms providing themselves a nitrogen source and in return producing various amino acids to maintain normal life activities for the hosts. Research has revealed that carbohydrates in forage can be fermented by intestinal microorganisms and the metabolites include certain short chain fatty acids (SCFAs) which can stimulate the intestinal mucosa, improve intestinal immunity, and promote energy absorption in the hosts [36, 37]. In our study, the concentration of carbohydrate metabolic pathways in TWAs 
had an advantage over NPDDs, which meant that microorganisms in the gut of TWAs could more efficiently promote cellulose and hemicellulose fermentation. As for energy metabolism, we found that the citrate cycle in TWAs was significantly more active than in NPDDs, indicating that the intestinal bacteria of TWAs could provide more energy for their own metabolic activities and were therefore beneficial for microorganism fermentation of forage in the intestine.

There are complex interactions in gut microbiota, such as mutualism, competition, prediction and commensalism [38]. Understanding the interactions and their responses to environmental is an important goal in ecology. However, defining a network structure in microbial community is especially challenging due to their vast diversity and as-yet uncultivated status. Thus, we used the network analysis to investigate the genus interactions according to their mutual competition (negative correlation) and mutual benefit (positive correlation). A positive correlation may relate to mutualism, commensalism or parasitism, while a negative correlation may result to competition, predation, etc [38]. In our network analysis, the TWAs were predominated by a higher positive correlations, while NPDDs were predominated by negative correlations. We speculated that plant-based diet in the gut of TWAs was more conductive to fermentation through the more corporation interactions than that of NPDD. In addition, we found that in TWAs, the total links, avgD and D were relatively higher than that of NPDDs, indicating that TWAs harbored a more complex ecological network, mostly also more metabolite. More complex interactions could build a more stable metabolic network environment for microbial population. Further study is needed to confirm this hypothesis.

A lower avgCC revealed that bacterial communities are largely composed of isolated nodes or loosely connected node groups, reflecting lower functional redundancy [39]. In our study, the avgCC in TWAs was relatively higher than NPDDs, indicating that the latter may harbor lower function redundancy than the former. Previous study had revealed that centralized nodes represent keystone species, which may influence the "information" flow among microbes [40]. In addition, these nodes play an important role in connecting many other microbes and are generally considered as important control points in the networks $A$ low level of $C B$, as a result of low frequency of centralized nodes [40].Thus, a higher CB in TWAs, indicated that the microorganism had different status in networks, implying that the lose of one or several keystone species may affect the stability of the whole bacterial community. Our result also showed that NPDDs harbored more key stone genus than TWA. Here, a total of three module hubs and six connectors were observed in TWAs (four connectors) and NPDDs (three module hubs and two connectors, respectively). However, none of them were the top 10 most abundant genus. The most abundant module microorganism OTU67 was at the 53th position, which indicated that the key stone genus in network not necessary the dominant microbes in the gut microbial community. However, the topological features are related to sequencing depth and different data processing. In our study, we only remained the OTUs that were present more than half of all samples, and this data processing may influence the topological features in bacteria network.

\section{Conclusion}

This study described the compositions, diversity and function of bacterial commubities in Tibetan wild assed and domestic donkeys. Based on 16s rRNA sequencing data, we found that the gut microbiota of TWAs was superior to that of NPDDs in bacterial community composition, function, and potentially high resistance to disease risk under similar forage nutrition intake. As the only wild species of perissodactyls on the Qinghai-Tibetan Plateau, the protection of Tibetan wild asses is of great significance to maintain the balance of the ecosystem and the harmony between human and animals. From the perspective of molecular biology, we studied the difference of gut microbiota between Tibetan wild asses and domestic donkeys. These results may help us understand the assembly of bacteria 
community in the gut of wild and domestic animals, and provide a theoretical basis for Tibetan wild asses' protection.

\section{Methods}

\section{Experimental design and sample collection}

In this study, all animal care procedures were consistent with the guidelines of Sanjiangyuan National Park Administration and Northwest Institute of Plateau Biology, CAS (NWIPB20160302).

For the feces collection of natural pasture domestic donkeys (NPDDs), the study was conducted in alpine meadow $\left(94^{\circ} 48^{\prime} \mathrm{E}, 34^{\circ} .99^{\prime} \mathrm{N}\right)$ at Menyuan County (Haibei Prefecture, Qinghai Province, China) with an altitude of $3200 \mathrm{~m}$ in August 2017. A total of 15 adult, females and healthy domestic donkeys (NPDDs) with familiar body conditions (385.43 $\pm 16.26 \mathrm{~kg}$ body weight, and not under current antibiotic treatment) were selected from a local herdsman. These laboratory donkeys had been grazing on natural pasture without supplementary feeding for two month (Jun to Jul). During the experiment, all laboratory animals grazed freely on the natural grassland and had free access to water. On the morning of Aug $16^{\text {th }}$, each donkey was attached to the grassland with a rope about 10 meters long, and the feeding range was kept about 15 meters apart. We numbered the 15 laboratory animals from 1 to 15 , and observed them in the corner where they were not easy to find. Once the fresh feces were discharged, according to the number, we collected them immediately and stored in sterile self-sealing bags with 4 degree portable refrigerator. In this way, all fresh feces that each donkey natural defecation were collected during the day. Finally, mixed the faces sufficiently, transformed into sterilized freezing tubes (approximately 2 milliliters each donkey) and stored in liquid nitrogen for later DNA extraction.

For the feces collection of Tibetan wild asses (TWAs), the study was conducted in alpine meadow $\left(101^{\circ} .06^{\prime} \mathrm{E}\right.$, $38^{\circ} .37^{\prime} \mathrm{N}$ ) at Qumarleb County (Yushu Prefecture, Qinghai Province, China) with an altitude of $4300 \mathrm{~m}$ in August 2017. This is a habitual area for Tibetan wild asses. On the morning of Aug $3^{\text {th }}$, we found a herd of 35 TWAs, through binoculars observation of the wild animals' behavior, we selected 15 adult, females and healthy TWAs as the laboratory animals. We used binoculars to observe the defecation of each wild ass, and 2 milliliters sterilized freezing tubes were used to quickly collect the mixed fresh feces and stored in liquid nitrogen. During the day, we walked about 20,000 steps and collected the feces of 15 experimental wild asses. During sampling, all laboratory wild animals had free access to water, no scared, no driven and antibiotic injection.

The forage samples were collected from quadrats $(50 \mathrm{~cm} \times 50 \mathrm{~cm})$ of grass on which the animals grazed. Twenty quadrats greater than $10 \mathrm{~m}$ apart were placed randomly to investigate the dominant species and collect the ground herbage. A total of 20 forage samples were collected from these two regions (10 per region). Forage samples were dried in a $65^{\circ} \mathrm{C}$ oven for $24 \mathrm{~h}$, then ground through a $1-\mathrm{mm}$ sieve and stored in a vacuum dryer for nutritional analysis.

\section{Determination of plant nutrient composition}

Total $\mathrm{N}$ was measured using the Kjeldahl method; the crude protein content was calculated as $6.25 \times \mathrm{N}$ (Method No. 984.13); the ether extract (EE) was measured using the Soxhlet system (Method No. 954.02); the acid detergent fiber (ADF) and neutral detergent fiber (NDF) were analyzed using the method described by Soest et al. (1991) [41]; the 
non-fibrous carbohydrate (NSC) was calculated as follows: NSC \% = 100-Ash\%-EE\%-NDF\%-CP(\%). The nutrition of the herbage and dominant species are shown in Table 1.

\section{DNA extraction and purification}

Microbial DNA was extracted from stool samples using the E. Z. N.A ${ }^{\circledR}$ Stool DNA Kit (Omega Bio-tek, Norcross, GA, USA) according to the manufacturer's protocols. DNA quality was assessed via $2 \%$ agarose gel electrophoresis and metagenomic DNA concentrations were determined with a NanoDrop ${ }^{\text {TM }} 2000$ (Thermo Fisher Scientific, Waltham, MA, USA). The $16 \mathrm{~S}$ rDNA V3-V4 regions of the ribosomal RNA gene were then amplified by PCR $\triangle 95^{\circ} \mathrm{C}$ for 2 min, followed by 27 cycles at $98{ }^{\circ} \mathrm{C}$ for $10 \mathrm{~s}, 62^{\circ} \mathrm{C}$ for $30 \mathrm{~s}$, and $68{ }^{\circ} \mathrm{C}$ for $30 \mathrm{~s}$, with a final extension at $68{ }^{\circ} \mathrm{C}$ for 10 min $\bigotimes$ using the following primers: 341F, CCTACGGGNGGCWGCAG; 806R, GGACTACHVGGGTATCTAAT. The 5'-end of 314F primer include 8-bp unique barcodes, which were used to split each sample. PCR reactions were performed in triplicate with a $50 \mu \mathrm{l}$ mixture containing $5 \mu \mathrm{l}$ of $10 \times \mathrm{KOD}$ buffer, $5 \mu \mathrm{l}$ of $2.5 \mathrm{mM}$ dNTPs, $1.5 \mu \mathrm{l}$ of each primer $(5 \mu \mathrm{m}), 1 \mu \mathrm{l}$ of KOD polymerase, and $100 \mathrm{ng}$ of template DNA. The PCR products were detected by electrophoresis on a $2 \%$ agarose gel and purified using the AxyPrep DNA Gel Extraction Kit (Axygen Biosciences, Union City, CA, USA) according to the manufacturer's instructions and quantified using QuantiFluor® -ST (Promega, Madison, WI, USA). The purified amplicons were then pooled equimolarly for paired-end sequencing on an Illumina (San Diego, CA, USA) HiSeq 2500 platform (Guangzhou Gene Denovo Biotechnology Co., Ltd., Guangzhou, China) using standard protocols.

\section{Sequencing and processing}

To get clean paired-end reads, the raw reads were filtered to remove reads containing greater than $10 \%$ unknown nucleotides (Ns) and fewer than $80 \%$ of base calls with quality scores less than 20 . FLASH version 1.2.11 was used to merge paired-end reads as raw tags with a minimum overlap of $10 \mathrm{bp}$ and mismatch error rates of 2\% [42]. The noisy raw tag sequences were filtered by QIIME to obtain high-quality, clean tags [43]. The clean tags then underwent chimera detection using the UCHIME algorithm until allchimeras were removed and effective tags were obtained [44]. All of the sequences were clustered into operational taxonomic units (OTUs) of " $\geq 97 \%$ " similarity using the UPARSE version 7.0.1001 pipeline [45] and the representative sequences were classified into organisms by applying a naive Bayesian model using the RDP classifier version 2.2 [46] based on the SILVA database [47].

\section{Statistical analysis}

Taxonomic composition for each cluster was evaluated at the phylum and genus level. Data related to bacterial community were statistically analyzed using SPSS version 17.0 (SPSS, Inc., Chicago, IL, USA). The significance of herbage nutritional composition and bacterial taxa was determined using the independent-samples $T$ test. The bacterial community function were predicted using Tax4Fun software [48]. The significant KEGG metabolic pathways were screened using STAMP software (http://kiwi.cs.dal.ca/Software/STAMP). The OTU rarefaction curves were calculated and plotted in QIIME. All alpha diversity was calculated in QIIME and graphed by Origin version 8.0 (OriginLab®, Northampton, MA, USA)). The principal coordinates analysis (PCoA) with unweighted UniFrac distance was plotted in $\mathrm{R}$ version 3.5.0. The unweighted pair-group method with arithmetic mean (UPGMA) classification tree was plotted by mothur version 1.41.1 [49]. Results are reported as means \pm SD. Effects were considered significant at $P<0.05$. 


\section{Network analysis and identification of putative keystone taxa in TWAs and NPDDs}

In order to understand the interaction of gut microbiota between TWA and NPDD, phylogenetic molecular ecological networks (pMENs) (http://ieg4.rccc.ou.edu/mena) were constructed based on Random Matrix Theory (RMT)-based methods. The protocols of network construction were described previously [50]. Briefly, only the OTUs that were presented in more than half of all samples were selected to in each TWAs and NPDDs gut microbiota genus. In order to compare the topological characteristics of bacteria network between TWAs and NPDDs, the pMENs were calculated with the same threshold (0.76). For each network, in above website, the window "global network properties" was used to calculate total nodes, total links, average degree (avgK), average clustering coefficient (avgCC), centralization of betweenness (CB) and density (D). The window "module separation and modularity calculation" was then used to calculate the value of within-module connectivity ( $\mathrm{Zi}$ ) and among modularity connectivity $(\mathrm{Pi})$. Finally, the visualization of these community co-occurrence networks was made with Cytoscape 3.3.0 [51].

A module typically contains many nodes, which are tightly connected to each other in one group, while have only few connection outside the group. The values of $\mathrm{Zi}$ and $\mathrm{Pi}$ are indicators of the connectivity of each node, and thus are often used to determine the topological role of these nodes [52]. According to the valises of $\mathrm{Zi}$ and $\mathrm{Pi}$, these nodes can be classified into four categories [53], including peripherrals (In the modules, the OTUs have few outside connections, $\mathrm{Pi}<0.62$ and $\mathrm{Zi}<2.5$ ), connectors (OTUs that connect modules, $\mathrm{Pi}>0.62$ ), network hubs (OTUs that highly connected within entire network, $\mathrm{Pi}>0.62$ and $\mathrm{Zi}>2.5$ ) and module hubs (OTUs that highly connected within modules, $\mathrm{Zi}>2.5$ ). Connectors, network hubs and module hubs were considered as the putative keystone taxa in a microbial community [54]. The keystone of gut microbiota in TWAs and NPDDs were identified based on above values.

\section{Abbreviations}

QTP: Qinghai Tibetan Plateau; TWAs: Tibetan wild assed; NPDDs: natural pasture domestic donkeys; Avgk: average degree; AvgCC: average clustering coefficient; $C B$ : centralization of betweenness; $D$ : density.

\section{Declarations}

\section{Acknowledgements}

We express our sincere gratitude to Prof Huan Li for his carefully review.

\section{Funding}

This work was supported by the National Key Research and Development Program of China (No. 2018YFD0502301), National Natural Science Foundation of China (No. 31402120), Key R\&D and transformation plan of Qinghai Province (No. 2019-SF-153), and "The Dawn of West China" 2018 Talent Training Program of CAS by Dongdong Chen. These funding bodies have the role of paying the composition materials used in this study and pay the salaries to students. None of these funding had any role in the design of the study and collection, analysis, data arrangement and in writing the manuscript. 


\section{Authors' contribution}

HJL, XQZ, SXX and LZ designed the experiments; HJJ performed the experiment; HJJ and LYH analyzed the data; HJL wrote the manuscript; LYH, TWX, NZ, XLZ, QL, DDC, FQH and XC helped to collect the faecal samples. All authors read and approved the final manuscript.

\section{Availability of data and materials}

The sequencing data for the 16S rRNA genes arepublicly available in the NCBI Short Read Archive (SRA) under accession NO. PRJNA553267.

\section{Ethic approval and consent to precipitate}

This study was carried out in accordance with the Guide and Use of Laboratory Animals, Ministry of Science and Technology of the People's Republic of China (2002). The protocol was approved by the Ethics and Welfare Committee of the Northwest Institute of Plateau Biology, Chinese Academy of Sciences (CAS), and the feces sample collection was permitted by Sanjiangyuan National Park Administration and Northwest Institute of Plateau Biology, CAS.

\section{Consent for population}

All authors have read and consent to the submission and publication of this manuscript.

\section{Competing interests}

The authors declare that they have no competing interests.

\section{Author details}

${ }^{a}$ Northwest Institute of Plateau Biology, Chinese Academy of Sciences, Xining, 810008, China.

${ }^{\mathrm{b}}$ Key Laboratory of Adaptation and Evolution of Plateau Biota, Chinese Academy of Sciences, Xining, 810008, China.

${ }^{c}$ University of Chinese Academy of Science, Beijing, 10049, China.

\section{References}

1.Wang CJ. Equus Kiang. Journal of Ecomic Animal 2014; 18.

2.Dong S, Wu X, Liu S, Su X, Wu Y, Shi J, Li X, Zhang X, Xu D, Weng J: Estimation of ecological carrying capacity for wild yak, kiang, and Tibetan antelope based on habitat suitability in the Aerjin Mountain Nature Reserve, China. Acta Ecol Sin 2015, 35(23):7598-7607. 
3.Guo X, Shao Q, Li Y, Wang Y, Wang D, Liu J, Fan J, Yang F: Application of UAV Remote Sensing for a Population Census of Large Wild Herbivores-Taking the Headwater Region of the Yellow River as an Example. Remote Sensing 2018, 10(7):1041.

4.MoehIman PDR: Equids: zebras, asses, and horses: status survey and conservation action plan: IUCN; 2002.

5.Sekirov I, Russell SL, Antunes LC, Finlay BB: Gut microbiota in health and disease. Physiol Rev 2010, 90(3):859904.

6.Hintz HF, Cymbaluk NF: Nutrition of the Horse. Annual Review of Nutrition 1994, 14(1):243.

7.0'Hara AM, Shanahan F: The gut flora as a forgotten organ. EMBO Rep 2006, 7(7):688-693.

8.Wardwell LH, Huttenhower C, Garrett WS: Current concepts of the intestinal microbiota and the pathogenesis of infection. Curr Infect Dis Rep 2011, 13(1):28-34.

9.Shepherd ML, Swecker WS, Jr., Jensen RV, Ponder MA: Characterization of the fecal bacteria communities of forage-fed horses by pyrosequencing of 16S rRNA V4 gene amplicons. FEMS Microbiol Lett 2012, 326(1):62-68.

10.Bian G, Ma S, Zhu Z, Su Y, Zoetendal EG, Mackie R, Liu J, Mu C, Huang R, Smidt H et al: Age, introduction of solid feed and weaning are more important determinants of gut bacterial succession in piglets than breed and nursing mother as revealed by a reciprocal cross-fostering model. Environ Microbiol 2016, 18(5):1566-1577.

11.Zhao JX, Zhang RS, Zhao YL, Du GF, MA ZY, Jiao JF. Analysis on Cecum Bacteria Diversity in Xinjiang Donkey. Grass-Feeding Livestock 2016; 4:20-25.

12.Gao H, Chi X, Qin W, Wang L, Song P, Cai Z, Zhang J, Zhang T: Comparison of the Gut Microbiota Composition between the Wild and Captive Tibetan Wild Ass (Equus kiang). J Appl Microbiol 2019.

13.Daniel H, Gholami AM, Berry D, Desmarchelier C, Hahne H, Loh G, Mondot S, Lepage P, Rothballer M, Walker A et al: High-fat diet alters gut microbiota physiology in mice. ISME J 2014, 8(2):295-308.

14.Liu CF, Zhang LZ, Fu HB, Li WQ, Zhang H, Li P et al. Delationship research between fecal microbes and short chain fatty acid. Acta Theriologica Sinica 2019;39:1-7.

15.Mariat D, Firmesse O, Levenez F, Guimaraes V, Sokol H, Dore J, Corthier G, Furet JP: The Firmicutes/Bacteroidetes ratio of the human microbiota changes with age. BMC Microbiol 2009, 9:123.

16.Middelbos IS, Vester Boler BM, Qu A, White BA, Swanson KS, Fahey GC, Jr.: Phylogenetic characterization of fecal microbial communities of dogs fed diets with or without supplemental dietary fiber using 454 pyrosequencing. PLoS One 2010, 5(3):e9768.

17.Sahu NP, Kamra DN: Microbial Eco-system of the Gastro-intestinal Tract of Wild Herbivorous Animals. Journal of Applied Animal Research 2002, 21(2):207-230.

18.Wu X, Zhang H, Chen J, Shang S, Wei Q, Yan J, Tu X: Comparison of the fecal microbiota of dholes highthroughput IIlumina sequencing of the V3-V4 region of the 16S rRNA gene. Appl Microbiol Biotechnol 2016, 100(8):3577-3586. 
19.Turnbaugh PJ, Ley RE, Mahowald MA, Magrini V, Mardis ER, Gordon JI: An obesity-associated gut microbiome with increased capacity for energy harvest. Nature 2006, 444(7122):1027-1031.

20.Ruth E. Ley PJT, Samuel Klein,, Gordon JI: Human gut microbes associated with obesity. Nature 2006(Vol444):1022-1023.

21.Xue D, Chen H, Zhao X, Xu S, Hu L, Xu T, Jiang L, Zhan W: Rumen prokaryotic communities of ruminants under different feeding paradigms on the Qinghai-Tibetan Plateau. Syst Appl Microbiol 2017, 40(4):227-236.

22.Li A, Jiang X, Wang Y, Zhang L, Zhang H, Mehmood K, Li Z, Waqas M, Li J: The impact of Bacillus subtilis 18 isolated from Tibetan yaks on growth performance and gut microbial community in mice. Microb Pathog 2019, 128:153-161.

23.Costa MC, Arroyo LG, Allen-Vercoe E, Stampfli HR, Kim PT, Sturgeon A, Weese JS: Comparison of the fecal microbiota of healthy horses and horses with colitis by high throughput sequencing of the V3-V5 region of the $16 S$ rRNA gene. PLoS One 2012, 7(7):e41484.

24.Qin J, Li Y, Cai Z, Li S, Zhu J, Zhang F, Liang S, Zhang W, Guan Y, Shen D et al: A metagenome-wide association study of gut microbiota in type 2 diabetes. Nature 2012, 490(7418):55-60.

25.Eckburg PB, Bik EM, Bernstein CN, Purdom E, Dethlefsen L, Sargent M, Gill SR, Nelson KE, Relman DA: Diversity of the human intestinal microbial flora. science 2005, 308(5728):1635-1638.

26.Paul B. Eckburg EMB, 2 Charles N. Bernstein,3 Elizabeth Purdom,4 Les Dethlefsen,2 Michael Sargent,3 Steven R. Gill,5 Karen E. Nelson,5 David A. Relman1,2,6*: Diversity of the Human Intestinal Microbial Flora. 2005.

27.Ludwig W, Euzéby J, Whitman WB: Taxonomic outlines of the phyla Bacteroidetes, Spirochaetes, Tenericutes (Mollicutes), Acidobacteria, Fibrobacteres, Fusobacteria, Dictyoglomi, Gemmatimonadetes, Lentisphaerae, Verrucomicrobia, Chlamydiae, and Planctomycetes. In: Bergey's Manualß of Systematic Bacteriology: Volume Four The Bacteroidetes, Spirochaetes, Tenericutes (Mollicutes), Acidobacteria, Fibrobacteres, Fusobacteria, Dictyoglomi, Gemmatimonadetes, Lentisphaerae, Verrucomicrobia, Chlamydiae, and Planctomycetes. Edited by Krieg NR, Staley JT, Brown DR, Hedlund BP, Paster BJ, Ward NL, Ludwig W, Whitman WB. New York, NY: Springer New York; 2010: 2124.

28.Belzer C, de Vos WM: Microbes inside-from diversity to function: the case of Akkermansia. ISME J 2012, 6(8):1449-1458.

29.Liu X, Fan H, Ding X, Hong Z, Nei Y, Liu Z, Li G, Guo H: Analysis of the gut microbiota by high-throughput sequencing of the V5-V6 regions of the 16S rRNA gene in donkey. Curr Microbiol 2014, 68(5):657-662.

30.Everard A, Belzer C, Geurts L, Ouwerkerk JP, Druart C, Bindels LB, Guiot Y, Derrien M, Muccioli GG, Delzenne NM et al: Cross-talk between <em>Akkermansia muciniphila</em> and intestinal epithelium controls diet-induced obesity. Proceedings of the National Academy of Sciences 2013, 110(22):9066-9071.

31.Human Microbiome Project C: Structure, function and diversity of the healthy human microbiome. Nature 2012, 486(7402):207-214.

32.Xiong J, Zhu J, Wang K, Wang X, Ye X, Liu L, Zhao Q, Hou M, Qiuqian L, Zhang D: The temporal scaling of bacterioplankton composition: high turnover and predictability during shrimp cultivation. Microbial ecology 2014, 
67(2):256-264.

33.Zeng S, Huang Z, Hou D, Liu J, Weng S, He J: Composition, diversity and function of intestinal microbiota in pacific white shrimp (Litopenaeus vannamei) at different culture stages. PeerJ 2017, 5:e3986.

34.Podgornaia Al, Laub MT: Determinants of specificity in two-component signal transduction. Curr Opin Microbiol 2013, 16(2):156-162.

35.Zhang LL, Wei XB, Li ZX, Hu QY, Si DY, Zhang RJ. The Role of Gut Bacteria of Monogastric Animals in Amino Acid Metabolism. Chinese Journal of Animal Science 2018,54(11),17-20.

36.El Kaoutari A, Armougom F, Gordon JI, Raoult D, Henrissat B: The abundance and variety of carbohydrate-active enzymes in the human gut microbiota. Nature Reviews Microbiology 2013, 11(7):497.

37.Jha R, Leterme P: Feed ingredients differing in fermentable fibre and indigestible protein content affect fermentation metabolites and faecal nitrogen excretion in growing pigs. Animal 2012, 6(4):603-611.

38.Faust K, Raes J: Microbial interactions: from networks to models. Nature Reviews Microbiology 2012, $10(8): 538$.

39.Sun MY, Dafforn KA, Johnston EL, Brown MV: Core sediment bacteria drive community response to anthropogenic contamination over multiple environmental gradients. Environ Microbiol 2013, 15(9):2517-2531.

40.Bissett A, Brown MV, Siciliano SD, Thrall PH: Microbial community responses to anthropogenically induced environmental change: towards a systems approach. Ecol Lett 2013, 16 Suppl 1:128-139.

41.Soest PJV, Robertson JB, Lewis BA: Methods for Dietary Fiber, Neutral Detergent Fiber, and Nonstarch Polysaccharides in Relation to Animal Nutrition. Journal of Dairy Science 1991, 74(10):3583-3597.

42.Magoc T, Salzberg SL: FLASH: fast length adjustment of short reads to improve genome assemblies. Bioinformatics 2011, 27(21):2957-2963.

43.Caporaso JG, Kuczynski J, Stombaugh J, Bittinger K, Bushman FD, Costello EK, Fierer N, Peña AG, Goodrich JK, Gordon JI et al: QIIME allows analysis of high-throughput community sequencing data. Nature Methods 2010, 7:335.

44.Edgar RC, Haas BJ, Clemente JC, Quince C, Knight R: UCHIME improves sensitivity and speed of chimera detection. Bioinformatics 2011, 27(16):2194-2200.

45.Edgar RC: UPARSE: highly accurate OTU sequences from microbial amplicon reads. Nature Methods 2013, 10.996.

46.Wang Q, Garrity GM, Tiedje JM, Cole JR: Naive Bayesian classifier for rapid assignment of rRNA sequences into the new bacterial taxonomy. Appl Environ Microbiol 2007, 73(16):5261-5267.

47.Pruesse E, Quast C, Knittel K, Fuchs BM, Ludwig W, Peplies J, Glockner FO: SILVA: a comprehensive online resource for quality checked and aligned ribosomal RNA sequence data compatible with ARB. Nucleic Acids Res 2007, 35(21):7188-7196.

48.Asshauer KP, Wemheuer B, Daniel R, Meinicke P: Tax4Fun: predicting functional profiles from metagenomic $16 S$ rRNA data. Bioinformatics 2015, 31(17):2882-2884.

Page 14/23 
49.Schloss PD, Westcott SL, Ryabin T, Hall JR, Hartmann M, Hollister EB, Lesniewski RA, Oakley BB, Parks DH, Robinson CJ et al: Introducing mothur: open-source, platform-independent, community-supported software for describing and comparing microbial communities. Appl Environ Microbiol 2009, 75(23):7537-7541.

50.Li H, Li T, Yao M, Li J, Zhang S, Wirth S, Cao W, Lin Q, Li X: Pika Gut May Select for Rare but Diverse Environmental Bacteria. Front Microbiol 2016, 7:1269.

51.Shannon P, Markiel A, Ozier O, Baliga NS, Wang JT, Ramage D, Amin N, Schwikowski B, Ideker T: Cytoscape: a software environment for integrated models of biomolecular interaction networks. Genome research 2003 , 13(11):2498-2504.

52.Deng Y, Jiang Y-H, Yang Y, He Z, Luo F, Zhou J: Molecular ecological network analyses. BMC bioinformatics 2012, 13(1):113.

53.Zhou J, Deng Y, Luo F, He Z, Yang Y: Phylogenetic molecular ecological network of soil microbial communities in response to elevated CO2. MBio 2011, 2(4).

54.Li H, Li T, Tu B, Kou Y, Li X: Host species shapes the co-occurrence patterns rather than diversity of stomach bacterial communities in pikas. Appl Microbiol Biotechnol 2017, 101(13):5519-5529.

\section{Tables}

Table 1 Comparison of the nutrient contents in the herbage composition of the TWAs and NPDDs groups

\begin{tabular}{lccc}
\hline Nutrient content of & \multicolumn{2}{c}{ Groups ${ }^{\mathrm{a}}$} & $\mathrm{P}$ \\
herbage (\%) & TWAs & NPDDs & 0.09 \\
\cline { 2 - 4 } $\mathrm{EE}^{\mathrm{b}}$ & $1.40 \pm 0.10$ & $1.32 \pm 0.02$ & 0.09 \\
\hline $\mathrm{CP}$ & $11.13 \pm 1.02$ & $10.96 \pm 0.43$ & 0.76 \\
\hline $\mathrm{ADF}$ & $22.51 \pm 1.02$ & $28.50 \pm 1.30$ & 0.44 \\
\hline $\mathrm{NDF}$ & $44.56 \pm 3.06$ & $48.82 \pm 1.87$ & 0.54 \\
\hline $\mathrm{NFC}$ & $27.80 \pm 3.00$ & $25.39 \pm 2.02$ & 0.03 \\
\hline $\mathrm{Ash}$ & $15.12 \pm 1.45^{\mathrm{a}}$ & $13.54 \pm 0.34^{\mathrm{b}}$ & Kobresia humilis, Kobresia \\
\hline Dominant species of & Kobresia tibetica, Kobresia & capillifolia & \\
\hline
\end{tabular}


a TWAs refers to Tibetan wild asses, NPDDs refers to natural pasture domestic donkeys.

$\mathrm{b}_{\mathrm{EE}}=$ ether extract, $\mathrm{CP}=$ crude protein, $\mathrm{ADF}=$ acid detergent fiber, $\mathrm{NDF}=$ neutral detergent fiber, NFC $=$ Non-fibrous carbohydrate.

Values in the same row with different superscripts are significantly different $(P<0.05)$.

Table 2 The taxonomic identification of putative keystone genus in network of TWAs and NPDDs bacterial community 


\begin{tabular}{|c|c|c|c|c|c|c|c|}
\hline $\begin{array}{l}\text { Species } \\
\mathrm{a}\end{array}$ & OTUID & $\begin{array}{c}\text { Average } \\
\text { relative } \\
\text { Abundance } \\
\mathrm{b}\end{array}$ & Taxnomy & $\begin{array}{c}\text { Abundance } \\
\text { ranking }{ }^{\mathrm{c}}\end{array}$ & Node type & $\overline{\mathrm{Pi}}$ & $\overline{Z i}$ \\
\hline \multirow{4}{*}{ TWAs } & OTU67 & 0.0662 & Unclassfied & 53th & Connector & 0.6914 & 0.4115 \\
\hline & OTU114 & 0.0046 & Unclassfied & 116th & Connector & 0.6950 & 2.2368 \\
\hline & OTU124 & 0.0034 & Oscillospira (Fir) & 121th & Connector & 0.7347 & 0.0000 \\
\hline & OTU134 & 0.0027 & Arthrobacter $(A c t)$ & 128th & Connector & 0.7160 & 1.7847 \\
\hline \multirow[t]{5}{*}{ NPDDs } & OTU58 & 0.0043 & Cellulosilyticum (Fir) & 118th & $\begin{array}{l}\text { Module } \\
\text { hubs }\end{array}$ & 0.5185 & 2.5628 \\
\hline & OTU112 & 0.0035 & $\begin{array}{c}\text { Erysipelatoclostridium } \\
\text { (Fir) }\end{array}$ & 126th & $\begin{array}{l}\text { Module } \\
\text { hubs }\end{array}$ & 0.4490 & 2.5019 \\
\hline & OTU118 & 0.0023 & $\begin{array}{l}\text { Bromus tectorum } \\
\text { (Cya) }\end{array}$ & 137th & $\begin{array}{l}\text { Module } \\
\text { hubs }\end{array}$ & 0.2449 & 2.9542 \\
\hline & OTU54 & 0.0517 & Anaerovibrio (Fir) & 68th & Connector & 0.6667 & 0.8452 \\
\hline & OTU114 & 0.0032 & Unclassfied & 130th & Connector & 0.6400 & 2.1243 \\
\hline
\end{tabular}

Fir Firmcutes, Act Actinobacteria, Cya, Cyanobacteria

a TWAs refers to Tibetan wild asses, NPDDs refers to natural pasture domestic donkeys.

$\mathrm{b}$ The average relative abundance of the OTUs in TWAs and NPDDs.

${ }^{\mathrm{C}}$ The rank of the OTUs for bacteria genus according to the order from high to low.

\section{Figures}



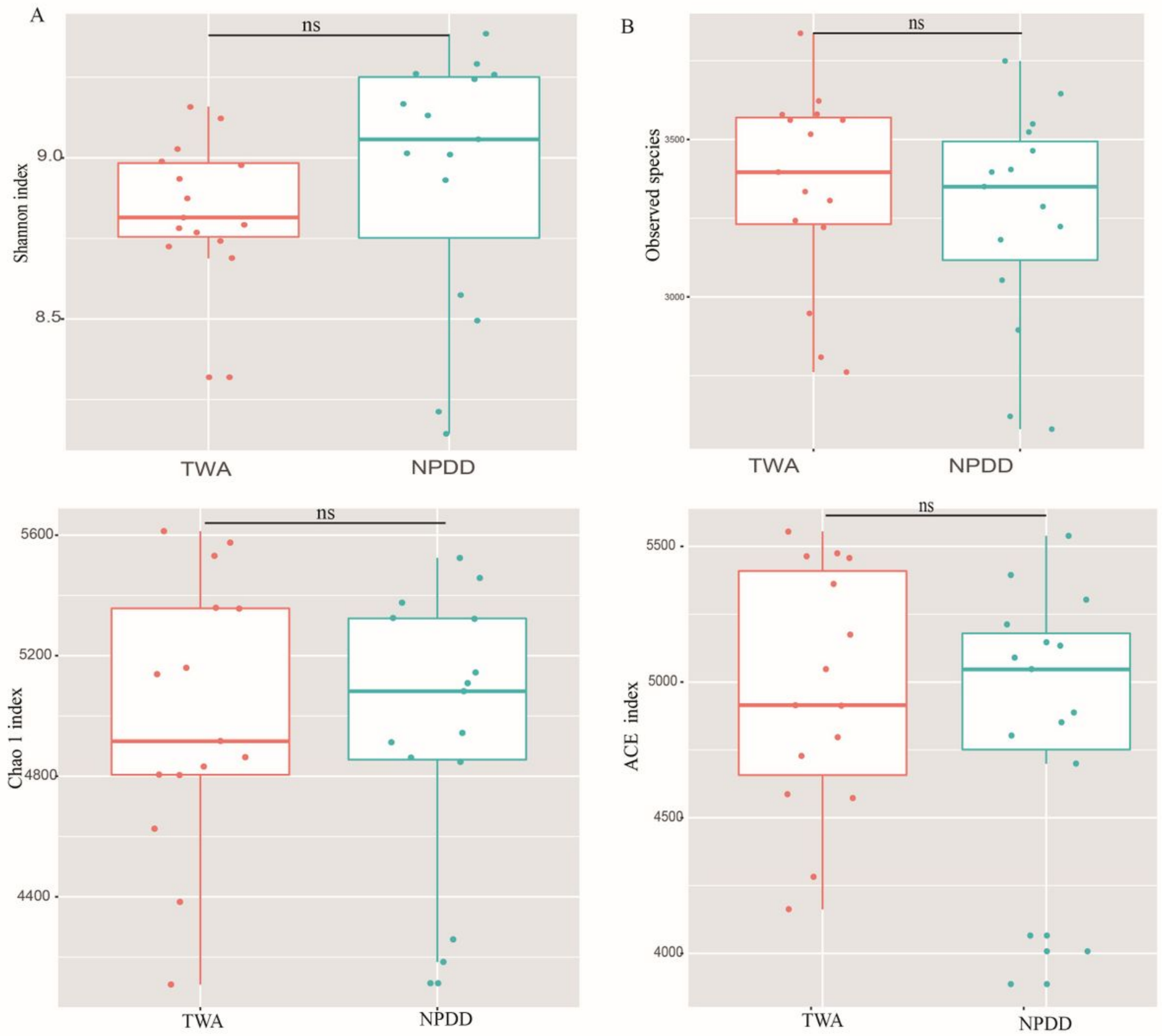

\section{Figure 1}

The statistical significance of alpha diversity estimators between TWAs and NPDDs. TWA=Tibetan wild asses, NPDD =natural pasture domestic donkeys. ${ }^{*} 0.01<p<0.05, * \star 0.001<P<0.01$, NS means no significant difference. 


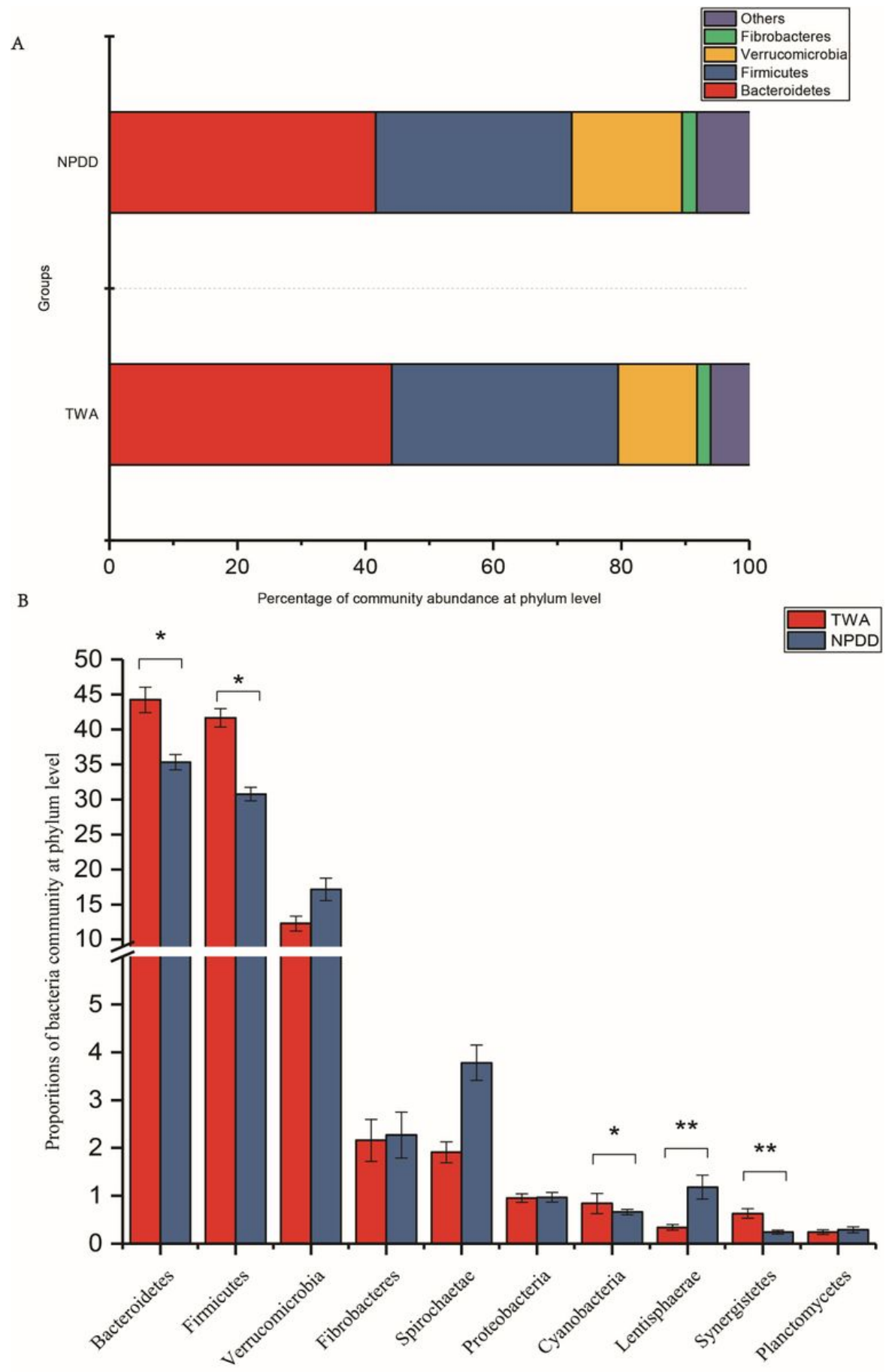

Figure 2

Bacterial community abundance at the phylum level of each group. (A) Bacterial community abundance bar plot at the phylum level. (B) Significance of the top 10 bacterial community abundance percentages at the phylum level. Each phylum that shares annotations was significantly different $(P<0.05)$. TWA=Tibetan wild asses, NPDD=natural pasture domestic donkeys. 
A
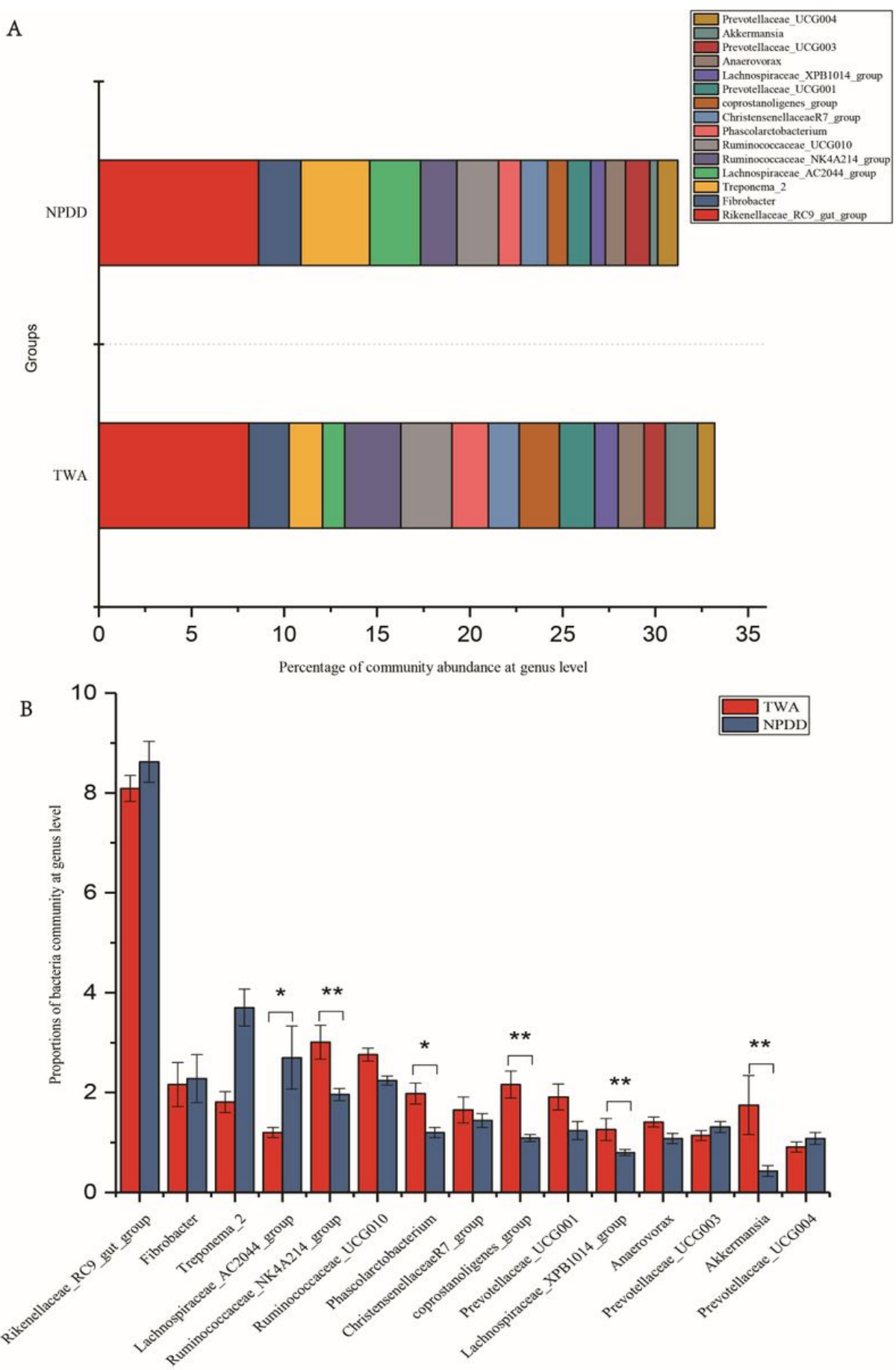

Figure 3

Bacterial community abundance at the genus level. (A) Bacterial community bar plot at the genus level. (B) Statistical significance of the top 15 bacterial community abundance percentages at the genus level. TWA=Tibetan wild asses, NPDD=natural pasture domestic donkeys. 
A

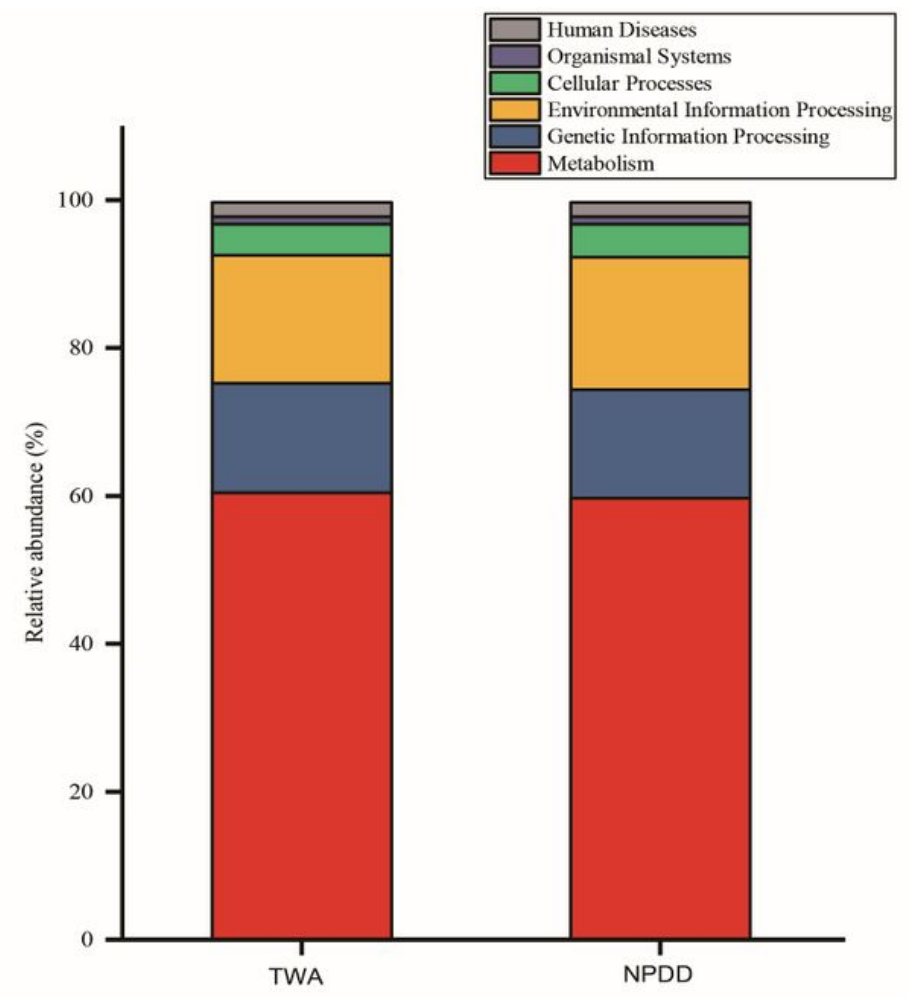

B

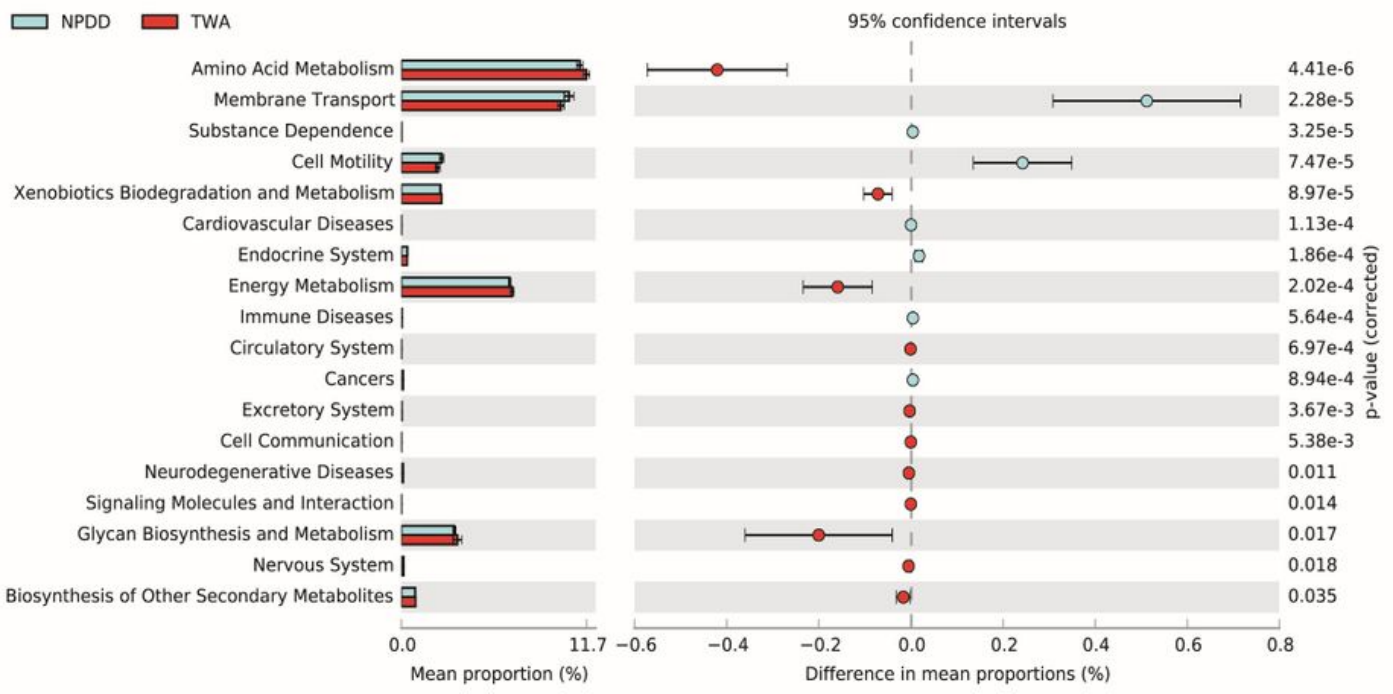

\section{Figure 4}

Metabolic pathway prediction at various KEGG levels between TWAs and NPDDs. (A) The six types of biological functional pathways in KEGG level 1. (B) The 17 significant metabolic pathways in KEGG level 2. TWA=Tibetan wild asses, NPDD=natural pasture domestic donkeys. 

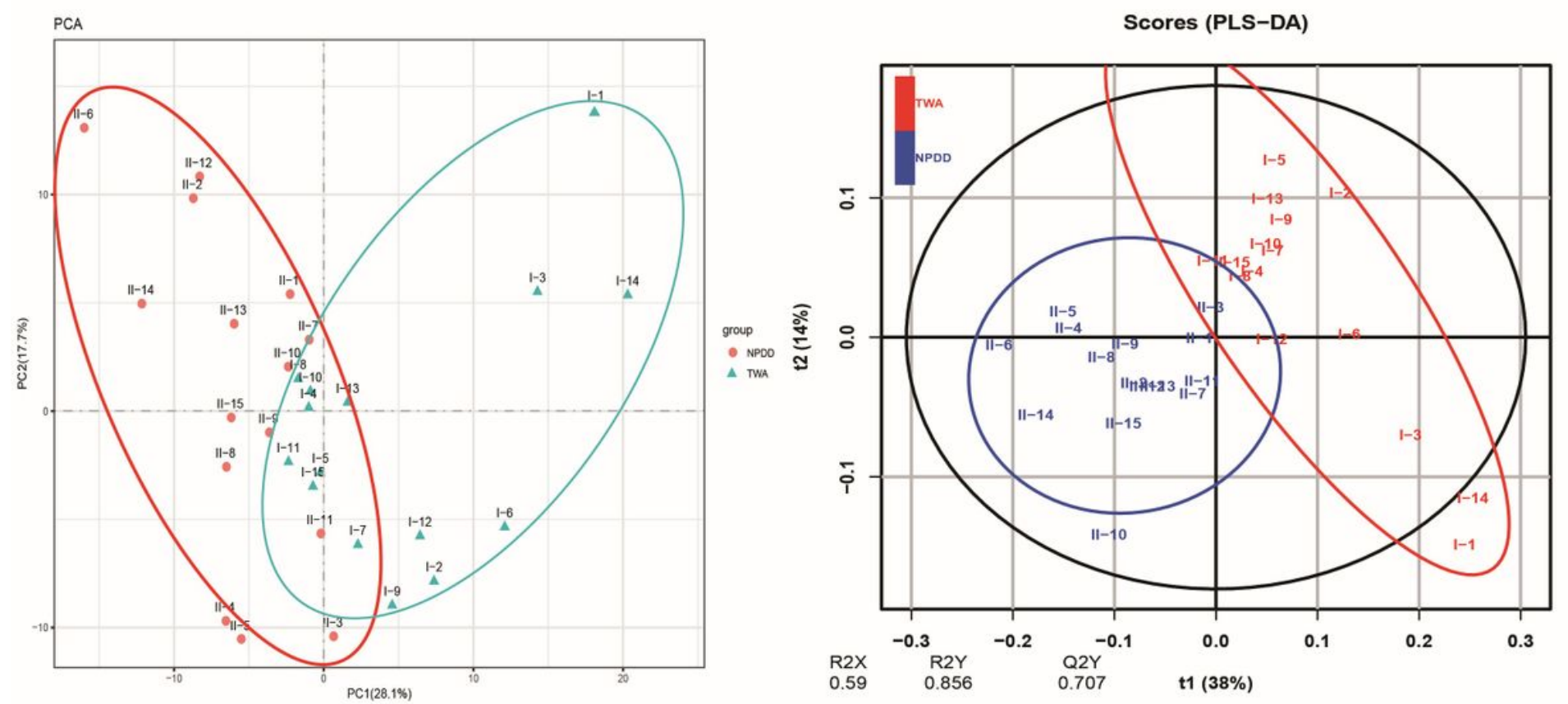

Figure 5

The principal component analysis (PCA) and partial least squares discriminant analysis (PLS-DA) of metabolite pathways between TWAs and NPDDs. TWA=Tibetan wild asses, NPDD=natural pasture domestic donkeys. 


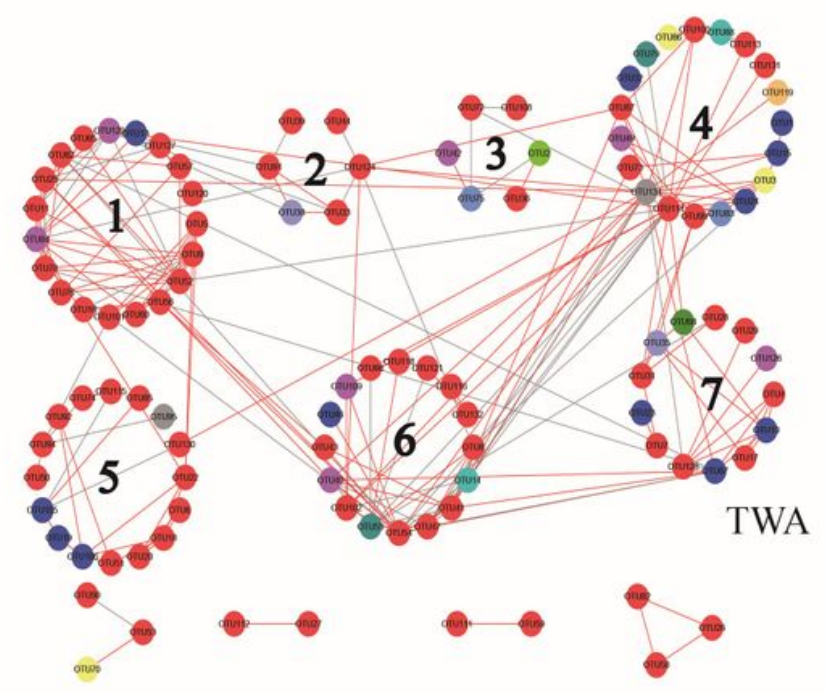

\begin{tabular}{lcc}
\hline Network Indexes & NPDD & TWA \\
\hline No.of samples & 15 & 15 \\
RMT cutoff & 0.76 & 0.76 \\
Total nodes & 89 & 105 \\
Total links & 104 & 207 \\
Positive links & 49 & 149 \\
Negative links & 55 & 58 \\
Average degree (avgK) & 2.34 & 3.94 \\
Average clustering coefficient (avgCC) & 0.12 & 0.27 \\
Centralization of betweeness (CB) & 0.235 & 0.305 \\
Density (D) & 0.027 & 0.038 \\
\hline
\end{tabular}

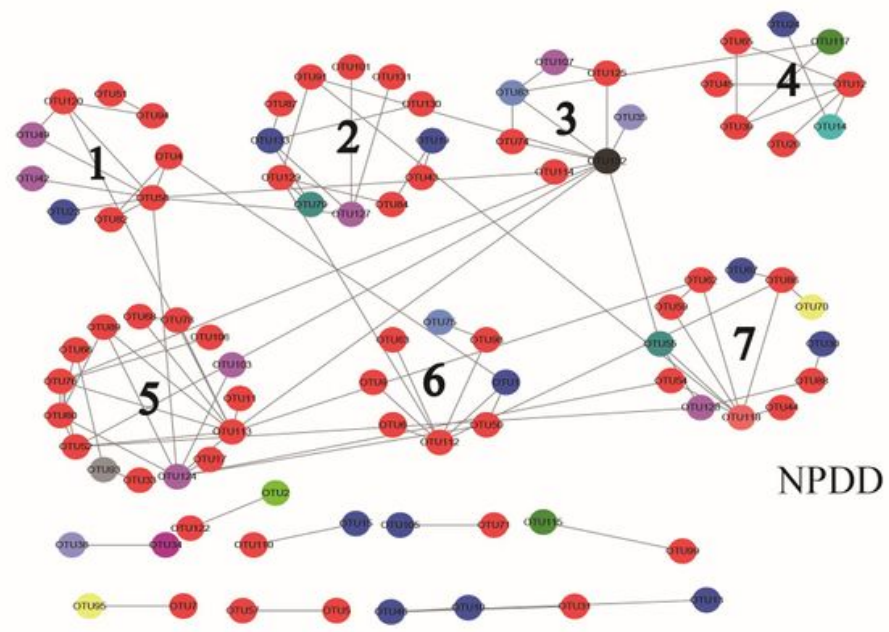

Bacteroidetes

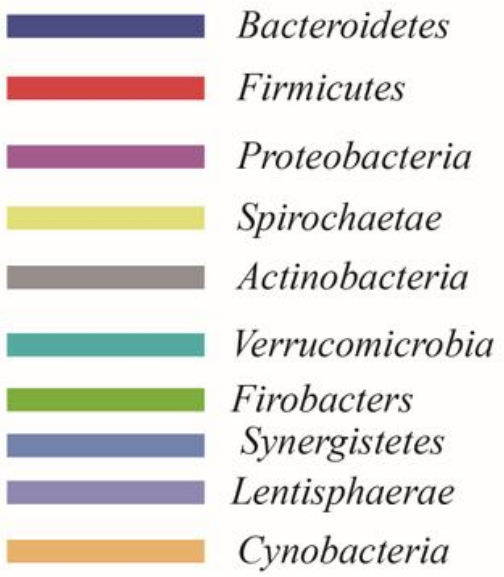

Figure 6

Comparision of gut bacterial networks between TWAs and NPDDs. Highly positive correlations is indicated by red color and negative correlations by gray color.TWA=Tibetan wild asses, NPDD=natural pasture domestic donkeys.

\section{Supplementary Files}

This is a list of supplementary files associated with this preprint. Click to download.

- Supplementarymaterials.docx 